\title{
A SIMPLIFIED PROOF OF THE ERDŌS-FUCHS THEOREM
}

\author{
D. J. NEWMAN ${ }^{1}$
}

\begin{abstract}
We reprove the theorem of Erdös and Fuchs in additive number theory. Whereas their solution rested on some special results in the $L^{2}$ theory of Fourier series, ours avoids these.
\end{abstract}

We present a variant of the proof of the very pretty theorem of Erdős and Fuchs [1]. Our proof is technically a bit simpler than theirs but, of more importance, it has the aesthetic advantage of sticking closer to the spirit of generating functions.

THEOREM (ERDós-FUCHS). Let $A$ be a set of nonnegative integers and denote by $r(n)$ the number of solutions to $n=a+a^{\prime}, a, a^{\prime} \in A$. If for some $C>0$, $\sum_{k=0}^{n}(r(k)-C)=O\left(n^{\alpha}\right)$, then $\alpha \geqslant \frac{1}{4}$.

Proof. If we write $A_{n}=\sum_{k=0}^{n}(r(k)-C)$ then we have

$$
\left(\sum z^{a}\right)^{2}=\frac{C}{1-z}+(1-z) \sum A_{n} z^{n}, \quad A_{n}=O\left(n^{\alpha}\right),
$$

Here, as later, we abbreviate our summation notation. It is to be generally understood that $a$ ranges over the set $A$, that $n$ ranges over the nonnegative integers, and, when we use the letter $b$, it will range over the nonnegative integers below $N$.

So let us multiply (1) by $\left(1+z+z^{2}+\cdots+z^{N-1}\right)^{2}, N>1$, and obtain thereby

$$
\left(\sum z^{a} \sum z^{b}\right)^{2}=\frac{C}{1-z}\left(\sum z^{b}\right)^{2}+\left(1-z^{N}\right) \sum z^{b} \cdot \sum A_{n} z^{n}
$$

which in turn gives the inequality

$$
\left|\sum z^{a} \sum z^{b}\right|^{2} \leqslant \frac{C N^{2}}{|1-z|}+2\left|\sum z^{b} \sum A_{n} z^{n}\right| .
$$

We now integrate this inequality around the circle $|z|=r, r<1$, with the measure of normalized arc length, i.e. $|d z| / 2 \pi r$.

First of all, setting $\sum z^{a} \cdot \sum z^{b}=\sum c_{n} z^{n}$, we observe that the $c_{n}$ are integers so that by Parseval's theorem we obtain

$$
\int\left|\sum z^{a} \sum z^{b}\right|^{2}=\sum c_{n}^{2} r^{2 n}>\sum c_{n} r^{2 n}=\sum r^{2 a} \cdot \sum r^{2 b}
$$

Received by the editors January 27, 1978.

AMS (MOS) subject classifications (1970). Primary 10A45, $10 \mathrm{~L} 05$.

Key words and phrases. Additive number theory, generating functions.

${ }^{1}$ Grant from NSF-MPS-75-08002. 
Furthermore if we assume, as we may, that $\alpha<1$ then the relation (1) tells us that, as $z \rightarrow 1^{-},\left(\sum z^{a}\right)^{2} \sim C /(1-z)$ and this insures the existence of a $\gamma>0$ for which $\left(\Sigma r^{2 a}\right)^{2}>\gamma /\left(1-r^{2}\right)$. Also we have $\Sigma r^{2 b}>N \cdot r^{2 N}$ and so (4) leads to the inequality

$$
\int\left|\sum z^{a} \sum z^{b}\right|^{2} \geqslant \sqrt{\frac{\gamma}{1-r^{2}}} \cdot N r^{2 N} .
$$

As to the right-hand side of (3) we first recall the elementary inequality

$$
\int \frac{1}{|1-z|}<1+\log \frac{1}{1-r^{2}}
$$

(obtained, for example, from the expansion for $(1-z)^{-1 / 2}$ ). Next we apply Schwarz' inequality to deduce that

$$
\begin{aligned}
\left(\int\left|\sum z^{b} \sum A_{n} z^{n}\right|\right)^{2} & <\int\left|\sum z^{b}\right|^{2} \cdot \int\left|\sum A_{n} z^{n}\right|^{2} \\
& =\sum r^{2 b} \cdot \sum A_{n}^{2} r^{2 n}<N \sum A_{n}^{2} r^{2 n} .
\end{aligned}
$$

Now $A_{n}=O\left(n^{\alpha}\right)$, and so, because of the elementary inequality $\Sigma n^{2 \alpha} r^{2 n}=$ $O\left(1 /\left(1-r^{2}\right)^{2 \alpha+1}\right)$ (obtained, for example, by comparison with the definite integral), we may conclude from (7) that

$$
2 \int\left|\sum z^{b}\right|\left|\sum A_{n} z^{n}\right|<\frac{A \sqrt{N}}{\left(1-r^{2}\right)^{\alpha+1 / 2}} .
$$

To complete the proof we choose $r^{2}=1-N^{-\lambda}, \lambda>1$, so that $r^{2 N}>(1-1 / N)^{N}>\left(1-\frac{1}{2}\right)^{2}=\frac{1}{4}$ and hence combining (3), (5), (6) and (8) gives

$$
(\sqrt{\gamma} / 4) N^{\lambda / 2+1}<C N^{2}(1+\lambda \log N)+A N^{1 / 2+\lambda \alpha+\lambda / 2},
$$

or

$$
\sqrt{\gamma} / 4<C N^{1-\lambda / 2}(1+\lambda \log N)+A N^{\lambda \alpha-1 / 2} .
$$

If, finally, we assume $0<\alpha<\frac{1}{4}$ then any choice of $\lambda$ which is above 2 but below $1 / 2 \alpha($ e.g. $\lambda=3 /(1+2 \alpha)$ ) makes both terms on the right side of (9) go to 0 as $N \rightarrow \infty$ and this contradiction completes the proof.

\section{REFERENCES}

1. P. Erdős and W. H. J. Fuchs, On a problem in additive number theory, J. London Math. Soc. 31 (1956), 67-73.

Department of Mathematics, Temple University, Philladelphia, Pennsyluvania 19122 